\title{
ON AN ESTIMATE FOR SOLUTIONS OF NONLINEAR ELLIPTIC VARIATIONAL INEQUALITIES ${ }^{1)}$
}

\author{
HARUO NAGASE
}

\section{Introduction}

Let $\Omega$ be a bounded domain in $R^{n}$ with the boundary $\partial \Omega$ of class $C^{0,1}$ and $E$ be a compact subset (resp. a compact subset on an $(n-1)$-dimensional hypersurface of class $\left.C^{0,1}\right)$ in $\Omega$. We assume that the usual function spaces $C^{k}(\bar{\Omega}), C_{0}^{k}(\Omega), L^{p}(\Omega), W^{1, p}(\Omega)$ and $W_{0}^{1, p}(\Omega)$ are known.

The first constraint condition is given by the following set:

$$
K_{1}=\left\{v \in W_{0}^{1, p}(\Omega) ; v(x) \in K(x) \text { a.e. (resp. p.p) } x \in E\right\},
$$

where $K(x)$ is a closed convex set in $R^{1}$ depending on $x$.

Next let $\partial_{1} \Omega$ and $\partial_{2} \Omega$ be two disjoint open subsets of $\partial \Omega$ such that $\partial \Omega=\overline{\partial_{1} \Omega} \cup \overline{\partial_{2} \Omega}$ and $\partial_{1} \Omega \neq \varnothing$. We set

$$
C_{(0)}^{1}(\bar{\Omega})=\left\{v \in C^{1}(\bar{\Omega}) ; v=0 \text { in a neighborhood of } \overline{\partial_{1} \Omega}\right\} .
$$

The completion of $C_{(0)}^{1}(\bar{\Omega})$ with respect to the norm $\|u\|_{1, p}=\|u\|_{p}+\|\nabla u\|_{p}{ }^{2)}$ is denoted by $W_{00}^{1, p}(\Omega)$. The following set $K_{2}$ defines the second constraint condition:

$$
K_{2}=\left\{v \in W_{(0)}^{1, p}(\Omega) ; v(x) \in \boldsymbol{k}(x) \text { p.p. } x \in \overline{\partial_{2} \Omega}\right\},
$$

where $k(x)$ is also a closed convex set in $R^{1}$ depending on $x$.

The aim of this paper is to establish an estimate for the solution $u \in K_{i}$ of the following variational inequality:

$$
\begin{gathered}
\sum_{j=1}^{n}\left\langle a_{j}(x, \nabla u), \partial x_{j}(u-v)\right\rangle+\left(a_{0}(x, u), u-v\right) \leqq(f, u-v) \\
\text { for any } v \in K_{i},
\end{gathered}
$$

Received March 24, 1986.

1) The content of this paper was lectured by the author at the Nonlinear P.D.E. Symposium, held at Tokyo University from February 24 to 26, 1986.

2) For the sake of simplicity we write $\|\cdot\|_{L^{p}(\Omega)}=\|\cdot\|_{p}$ and $\left(\partial_{1} u, \partial_{x_{2}} u, \cdots, \partial_{x_{n}} u\right)=\nabla u$. 
where we describe the pairing between $W_{0}^{1, p}(\Omega)\left(W_{(0)}^{1, p}(\Omega)\right)$ and its dual by $\langle$,$\rangle and the inner product of L^{2}(\Omega)$ by $($,$) .$

At first we note that the norm $\|u\|_{1, p}$ is equivalent to the norm $\|\nabla u\|_{p}$ in $W_{0}^{1, p}(\Omega)$ or $W_{(0)}^{1, p}(\Omega)$ by Poincaré's inequality.

In the case when $K=K_{1}$ (resp. $K=K_{2}$ ) and $E=\Omega\left(\right.$ resp. $\left.\partial_{1} \Omega=\varnothing\right)$, many authors obtained a number of results with respect to the properties of solutions of (3) for operators $a_{j}$ and $K(x)$ (resp. $k(x)$ ) of various types. Here we do not refer explicitly to such cases.

Before stating our theorem, we will refer to results related to our problems. D. Kinderlehrer ([23]) proved the existence of a Lipschitz continuous solution in $R^{2}$ under the assumption that $a_{i}=a_{j}(\eta)^{3)}$ satisfies $\sum_{j=1}^{2}\left(a_{j}(\eta)-a_{j}\left(\eta^{\prime}\right)\right)\left(\eta_{j}-\eta_{j}^{\prime}\right) \geqq \nu\left|\eta-\eta^{\prime}\right|^{2}(\nu>0)$ and $E$ is a segment. In [13] J. Frehse discussed the continuity of the first order derivatives of solutions when $a_{j}$ satisfies $\sum_{i, j=1}^{n}\left(\partial \alpha_{j} / \partial \eta_{i}\right)(\eta) \xi_{i} \xi_{j} \geqq \nu|\xi|^{2}(\nu>0)$ and $E$ is an $(n-1)$-dimensional manifolds. Also G. H. Williams ([38]) proved the existence of a Lipschitz continuous solution for the case that $a_{j}$ satisfies the inequality $\sum_{j=1}^{n}\left(a_{j}(x, z, \eta)-a_{j}\left(x, z^{\prime}, \eta^{\prime}\right)\right)\left(\eta_{j}-\eta_{j}^{\prime}\right)+\left(a_{0}(x, z, \eta)-a_{0}\left(x, z^{\prime}, \eta^{\prime}\right)\right)\left(z-z^{\prime}\right) \geqq$ $C\left|\eta-\eta^{\prime}\right|^{2}(C>0)$ and $E$ is a subset in $\Omega$. In the above three cases $K(x)$ is only of the type $K(x)=\{u(x) \geqq \phi(x)\}$ for a given function $\phi(x)$. Additionally when $n=2$ and $\partial_{2} \Omega=(a, b) \times\left\{x_{2}=0\right\}$ particularly, D. Kinderlehrer ([24]) showed that solutions belong to class $C^{1, \alpha}\left(\Omega \cup \partial_{2} \Omega\right)$ under the same condition on $a_{j}$ as in [23] and with the assumption that $k(x)=\{u(x) \geqq \psi(x)\}$ for some function $\psi(x)$.

Secondly we mention results for the cases when $a_{i}$ degenerate. To begin with we impose the next conditions on $a_{j}(j=0,1, \cdots, n)$ :

$$
\left\{\begin{array}{l}
\sum_{j=1}^{n} a_{j}(x, z, \eta) \eta_{j} \geqq a|\eta|^{p}-b(x)|z|^{p}-l(x), \\
\left|a_{j}(x, z, \eta)\right| \leqq a^{\prime}|\eta|^{p-1}+g(x)|z|^{p-1}+h(x), \quad j=1, \cdots, n \\
\left|a_{0}(x, z, \eta)\right| \leqq d(x)|\eta|^{p-1}+e(x)|z|^{p-1}+m(x) .
\end{array}\right.
$$

Assuming that $a_{j}(j=1, \cdots, n)$ are independent of $z$ and that $b, g, h$, and $l$ equal to zero and that furthermore $a_{0}=|z|^{p-2} z$, H. Beirão da Veiga ([35]) obtained the $L^{\infty}(\Omega)$ estimate for solutions of variational inequalities under the constraint condition that $u(x) \geqq \phi_{i}(x)$ on $E_{i}(i=1, \cdots, m)$. In [35] the boundary condition is of either Dirichlet, Neumann or Signorini's

3) In place of the operator $a_{j}(x, u, \nabla u)$ we consider the function $a_{j}(x, z, \eta)$ defined on $\Omega \times R^{1} \times R^{n}$. 
type, furthermore the mixed type of these conditions is also treated. Moreover in [36] he established the $L^{\infty}(\Omega)$ estimate and the $C^{\alpha}(\bar{\Omega})$ estimate under the assumption (4) for the constraint condition $K=\left\{v \in W^{1, p}(\Omega) ; v(x) \geqq\right.$ $\psi(x)$ on $\partial \Omega, v(x)=\psi(x)$ on $\Gamma\}$. H. Beirão da Veiga-F. Conti ([37]) proved that solutions belong to $C^{\alpha}(\bar{\Omega})$ when $a_{j}(j=1, \cdots, n)$ are independent of $z$ and $a_{0}=0$ in (4), in addition $a_{j}$ satisfies the condition $\sum_{j=1}^{n}\left(a_{j}(x, \eta)-\right.$ $\left.a_{j}\left(x, \eta^{\prime}\right)\right)\left(\eta_{j}-\eta_{j}^{\prime}\right)>0$. Here the constraint condition is of type: $u(x) \geqq \phi(x)$ on E. When $b=e=g=0$ in (4), A. Domarkas ([9], [10]) showed that solutions belong to $C^{\alpha}(\bar{\Omega})$ for the constraint condition $K: K=\left\{v \in W_{0}^{1, p}(\Omega)\right.$; $v(x) \geqq \phi_{1}(x)$ on $E_{1}$ and $v(x) \leqq \phi_{2}(x)$ on $\left.E_{2}\right\}$ or $K=\left\{v \in W^{1, p}(\Omega) ; v(x) \geqq \psi_{1}(x)\right.$ on $\Gamma_{1}$ and $v(x) \leqq \psi_{2}(x)$ on $\left.\Gamma_{2}\right\}$. In the above works $E$ and $E_{i}$ are subsets of $\Omega$ and $\Gamma, \Gamma_{i}$ are subsets of $\partial \Omega$, additionally $\phi_{i}(x), \psi(x)$ and $\psi_{i}(x)$ are some given functions.

Throughout this paper let $p \geqq 2$ and let us assume that $\alpha \geqq 0$ if $p \geqq n$, $0 \leqq \alpha<n(p-1) /(n-p)-1$ if $p<n$.

Now we impose the following assumptions on $a_{\jmath}(j=0,1, \cdots, n)$ :

Assumption A.

(I) (i) $a_{j}=a_{j}(x, \eta) \in C^{0}\left(\Omega \times R^{n}\right) \cap C^{1}\left(\Omega \times\left(R^{n}-\{\mathbf{0}\}\right)\right), a_{j}(x, \mathbf{0})=0$, $j=1, \cdots, n$.

(ii) $\sum_{i, j=1}^{n} \frac{\partial a_{j}}{\partial \eta_{i}}(x, \eta) \xi_{i} \xi_{j} \geqq \gamma\left(\kappa_{0}+|\eta|^{p-2}\right)|\xi|^{2}$,

$\left|\frac{\partial a_{j}}{\partial \eta_{i}}(x, \eta)\right| \leqq \Lambda\left(\kappa_{0}+|\eta|^{p-2}\right), \quad i=1, \cdots, n$,

$\left|\frac{\partial a_{j}}{\partial x_{k}}(x, \eta)\right| \leqq \Lambda\left(\kappa_{0}|\eta|+|\eta|^{p-1}\right), \quad k=1, \cdots, n$.

(II) (i) $a_{0}=a_{0}(x, z) \in C^{0}\left(\Omega \times R^{1}\right) \cap C^{1}\left(\Omega \times\left(R^{1}-\{0\}\right)\right), a_{0}(x, 0)=0$.

(ii) $\frac{\partial a_{0}}{\partial z}(x, z) \geqq 0, \quad\left|\frac{\partial a_{0}}{\partial z}(x, z)\right| \leqq \Lambda\left(|z|^{\alpha}+1\right)$,

$\left|\frac{\partial a_{0}}{\partial x_{k}}(x, z)\right| \leqq \Lambda\left(|z|^{\alpha+1}+1\right), \quad k=1, \cdots, n$.

in the above $x \in \Omega, z \in R^{1}-\{0\}, \eta \in R^{n}-\{0\}$ and $\xi \in R^{n}$. And $\kappa_{0}$ is a nonnegative constant, $\gamma$ and $\Lambda$ are some positive constants.

Hereafter we write by the same $C$ all constants independent of $u$ and $f$, which appear in (3). We define the following function $\Phi(x)$ in $R^{n}$ :

$$
\Phi(x)= \begin{cases}\operatorname{dis}(x, E \cup \partial \Omega) & \text { for the case of } K=K_{1} \\ \operatorname{dis}(x, \partial \Omega) & \text { for the case of } K=K_{2}\end{cases}
$$


where $\operatorname{dis}(A, B)$ is the distance between $A$ and $B$.

Our theorem is as follows.

THEOREM. Under the assumption $A$ if $f \in W^{1, p^{*}}(\Omega)$ (resp. $L^{2}(\Omega)$ ) in the case of $\kappa_{0}=0$ (resp. $\kappa_{0} \neq 0$ ), the following assertions hold for the solution $u$ of the nonlinear variational inequality (3).

( I ) In any case $\Phi(x) a_{j}(x, \nabla u) \in W^{1, p^{*}}(\Omega), \Phi(x)|\nabla u|^{p / 2} \in W^{1,2}(\Omega), j=1, \cdots, n$.

(II) If $\kappa_{0}=0$,

$$
\left\|\Phi(\cdot) a_{j}(\cdot, \nabla u)\right\|_{1, p^{*}}^{p^{*}}+\left\|\Phi(\cdot)|\nabla u|^{p / 2}\right\|_{1,2}^{2} \leqq C\left(1+\|f\|_{1, p^{*}}^{p^{*}}+\|f\|_{p^{*}}^{(\alpha+2) /(p-1)}\right) .
$$

(III) If $\kappa_{0} \neq 0, \Phi(x) \nabla u \in W^{1,2}(\Omega)$ and

$$
\|\Phi(\cdot) \nabla u\|_{1,2}^{2}+\left\|\Phi(\cdot) a_{j}(\cdot, \nabla u)\right\|_{1, p^{*}}^{p^{*}}+\left\|\Phi(\cdot)|\nabla u|^{p / 2}\right\|_{1,2}^{2} \leqq C\left(1+\|f\|_{2}^{2 \beta / p}\right),
$$

where $\beta=\max (p, \alpha+2)$ and $p^{*}$ is the dual number of $p$, i.e., $1 / p+1 / p^{*}$ $=1$.

In connection to the estimate in our theorem the following results were obtained by G. H. Yakovlev. He gave first the estimate of $\left\|\partial_{x_{j}}\left(\left|\partial_{x_{i}} u\right|^{p / 2}\right)\right\|_{L^{2}\left(\Omega_{\delta}\right)}$ for solutions of the variational problem which is derived from the functional of the form $I(v)=\int_{\Omega}\left\{\sum_{j=1}^{n}\left|\partial_{x_{j}} v\right|^{p}+a(x) v\right\} d x$ with the Dirichlet boundary condition ([40]). Here $\Omega_{\delta}=\{x \in \Omega$; $\operatorname{dis}(x, \partial \Omega)>\delta\}$.

In [43] also he obtained estimates of

$$
\left\|a_{j}(\cdot, u, \nabla u)\right\|_{W^{1}, p^{*}\left(\Omega_{j}\right)} \quad \text { and } \quad\left\|\frac{\partial a_{j}}{\partial x_{k}}(\cdot, u, \nabla u)\right\|_{W^{1}, p^{*}\left(\Omega_{j}\right)}
$$

for weak solutions of the nonlinear elliptic equation $\sum_{j=1}^{n} \partial_{x_{j}}\left(a_{j}(x, u, \nabla u)\right)$ $=a_{0}(x, u, \nabla u)$ under weaker assumptions than ours. The method in [43] is to use the quotient of differentials with the usual parallel transfor. mation, namely,

$$
h^{-1}\left[u\left(x_{1}, x_{2}, \cdots, x_{i-1}, x_{i}+h, x_{i+1}, \cdots, x_{n}\right)-u(x)\right],
$$

which implies that the estimate of the norm is obliged to be restricted in $\Omega_{\delta}$. In this paper we use a transformation with weight function $\Phi(x)$, so we can estimate the norm in the whole $\Omega$. Our estimates are very similar to that of G.H. Yakovlev ([41]), where a nonlinear elliptic equation with the Dirichlet boundary condition was treated. He prepared the estimate for smooth solutions and applied the Galerkin's method, so his technique can not be applied to our variational inequality.

Finally we refer to the regularity of weak soultions of the nonlinear elliptic equation. Let us put $v=u \pm \phi$ in (3) for a solution $u$ and any function $\phi$ in $C_{0}^{\infty}(\Omega \backslash E)$ (resp. $\left.C_{0}^{\infty}(\Omega)\right)$ in the case of $K=K_{1}$ (resp. $K=K_{2}$ ), 
then we see that $u$ is a weak solution of the nonlinear elliptic equation

$$
-\sum_{j=1}^{n} \partial_{x_{j}}\left(a_{j}(x, \nabla u)\right)+a(x, u)=f
$$

in $\Omega \backslash E($ resp. $\Omega)$.

J. C. Evans ([11]) and J. L. Lewis ([26]) proved that weak solutions of (5) belong to $C_{\mathrm{loc}}^{1, \alpha}(\Omega \backslash E)$ (resp. $C_{\mathrm{loc}}^{1, \alpha}(\Omega)$ ) when $a_{j}=|\nabla u|^{p-2} \partial_{x_{j}} u$ and $a_{0}=0$. Besides E. DiBenedetto ([8]) and P. Tolksdorf ([33]) showed the same results under weaker assumptions on $a_{j}$ and $a_{0}$ than those of [11] and [26].

1.

The existence of a unique solution for the variational inequality (3) is derived from Lemma 1.2 in this section.

First we will prepare the following lemma which will be frequently used in this paper:

LEMMA 1.1. Under the assumption A the following assertions hold:

(i) $a_{j}(j=0,1, \cdots, n)$ are estimated in such a way that

$$
\begin{aligned}
& \left|a_{j}(x, \eta)\right| \leqq C\left(\kappa_{0}+|\eta|^{p-2}\right)|\eta|, \quad j=1, \cdots, n, \\
& \left|a_{0}(x, z)\right| \leqq C\left(1+|z|^{\alpha}\right)|z| .
\end{aligned}
$$

(ii) (P. Tolksdorf [33, p. 129] and P. Lindqvist [28, p. 310]) There exists a positive number $\gamma_{0}$ depending only on $\gamma$ and $p$ such that

$$
\begin{aligned}
& \sum_{j=1}^{n}\left(a_{j}(x, \eta)-a_{j}\left(x, \gamma^{\prime}\right)\right)\left(\eta_{j}-\eta_{j}^{\prime}\right) \\
& \geqq \gamma_{0}\left(\kappa_{0}+|\eta|^{p-2}+\left|\eta^{\prime}\right|^{p-2}\right)\left|\eta-\eta^{\prime}\right|^{2}, \\
& \left(a_{0}(x, z)-a_{0}\left(x, z^{\prime}\right)\right)\left(z-z^{\prime}\right) \geqq 0 .
\end{aligned}
$$

Here $x \in \Omega, \eta, \eta^{\prime} \in R^{n}$ and $z, z^{\prime} \in R^{1}$.

Proof.

(i) The estimates (1.1) and (1.2) are derived from (I)-(ii) and (II)-(ii) in the assumption $\mathrm{A}$ and the equalities

$$
a_{j}(x, \eta)=\int_{0}^{1} \sum_{i=1}^{n} \frac{\partial a_{j}}{\partial \eta_{i}}(x, t \eta) \eta_{i} d t, \quad a_{0}(x, z)=\int_{0}^{1} \frac{\partial a_{0}}{\partial z}(x, t z) z d t
$$

(ii) Without loss of the generality we may assume that $|\eta| \leqq\left|\eta^{\prime}\right|$. According to simple calculations and (I)-(ii) in the assumption A, we see that 


$$
\begin{aligned}
& \sum_{j=1}^{n}\left(a_{j}(x, \eta)-a_{j}\left(x, \eta^{\prime}\right)\right)\left(\eta_{j}-\eta_{j}^{\prime}\right) \\
& \quad=\sum_{j=1}^{n} \int_{0}^{1} \frac{d}{d t} a_{j}\left(x, t \eta+(1-t) \eta^{\prime}\right)\left(\eta_{j}-\eta_{j}^{\prime}\right) d t \\
& \quad \geqq \int_{0}^{1 / 4} \sum_{i, j=1}^{n} \frac{\partial a_{j}}{\partial \eta_{i}}\left(x, t \eta+(1-t) \eta^{\prime}\right)\left(\eta_{i}-\eta_{i}^{\prime}\right)\left(\eta_{j}-\eta_{j}^{\prime}\right) d t \\
& \quad \geqq \gamma \int_{0}^{1 / 4}\left(\kappa_{0}+\left|t \eta+(1-t) \eta^{\prime}\right|^{p-2}\right)\left|\eta-\eta^{\prime}\right|^{2} d t .
\end{aligned}
$$

On the other hand it holds that

$$
\begin{aligned}
\mid t \eta+ & (1-t) \eta^{\prime}|\geqq(1-t)| \eta^{\prime}|-t| \eta \mid \\
& =(3 / 4-t)\left|\eta^{\prime}\right|-(t+1 / 4)|\eta|+1 / 4\left(\left|\eta^{\prime}\right|+|\eta|\right) \\
& \geqq(t+1 / 4)\left(\left|\eta^{\prime}\right|-|\eta|\right)+1 / 4\left(\left|\eta^{\prime}\right|+|\eta|\right) \\
& \geqq 1 / 4\left(\left|\eta^{\prime}\right|+|\eta|\right),
\end{aligned}
$$

for all $t \in[0,1 / 4]$. The inequalities (1.5) and (1.6) imply (1.3). The estimate (1.4) can be proved more easily.

Q.E.D.

Remark. In (1.6) we note that the estimate $\left|t \eta+(1-t) \eta^{\prime}\right| \geqq(1 / 4)$. $\left|\eta^{\prime}-\eta\right|$ holds for all $t \in[0,1 / 4]$. Thus we have the following estimate in place of (1.3):

$$
\sum_{j=1}^{n}\left(a_{j}(x, \eta)-a_{j}\left(x, \eta^{\prime}\right)\right)\left(\eta_{j}-\eta_{j}^{\prime}\right) \geqq \gamma_{0}\left(\kappa_{0}+\left|\eta-\eta^{\prime}\right|^{p-2}\right)\left|\eta-\eta^{\prime}\right|^{2} .
$$

This is due to K. L. Kuttler Jr. ([25]).

Lemma 1.2. For $u, v \in W_{0}^{1, p}(\Omega)\left(W_{(0)}^{1, q}(\Omega)\right)$ we define the operator $A$ in such a way that $(A(u), v)=\sum_{j=1}^{n}\left\langle a_{j}(x, \nabla u), \partial_{x_{j}} v\right\rangle+\left(a_{0}(x, u), v\right)$, then the operator $A$ is pseudo-monotone and coercive from $W_{0}^{1, p}(\Omega)\left(W_{(0)}^{1, p}(\Omega)\right)$ to its dual space.

Proof. We write $W_{0}^{1, p}(\Omega)\left(W_{(0)}^{1, p}(\Omega)\right)$ simply by $V$ and its dual space by $V^{\prime}$.

In order to prove the pseudo-monotonicity of the operator $A$, it is enough to show that the operator $A$ is (i) bounded, (ii) hemi-continuous and (iii) monotone (J.L. Lions [29], p. 179).

(i) Boundedness.

According to the definition

$$
|(A(u), v)| \leqq \sum_{j=1}^{n}\left|\left\langle a_{j}(x, \nabla u), \partial_{x_{j}} v\right\rangle\right|+\left|\left(a_{0}(x, u), v\right)\right| .
$$


We estimate each term on the right-hand side of (1.8).

At first we obtain

$$
\begin{aligned}
\left|\left\langle a_{j}(x, \nabla u), \partial_{x_{j}} v\right\rangle\right| & \leqq C \int_{\Omega}\left(\kappa_{0}+|\nabla u|^{p-2}\right)|\nabla u| \cdot|\nabla v| d x \\
& \leqq C\left(\|\nabla u\|_{p^{*}}\|\nabla v\|_{p}+\|\nabla u\|_{p}^{p-1}\|\nabla v\|_{p}\right),
\end{aligned}
$$

by (1.1) and Hölder's inequality. Since $p \geqq 2, p$ and $p^{*}$ satisfy $1<p^{*} \leqq$ $2 \leqq p$. Thus the inequality $\|\nabla u\|_{p^{*}} \leqq C\|\nabla u\|_{p}$ is verified. Therefore from (1.9) we deduce the estimate

$$
\left|\left\langle a_{j}(x, \nabla u), \partial_{x_{j}} v\right\rangle\right| \leqq C\left(\|u\|_{V}+\|u\|_{V}^{p-1}\right)\|v\|_{V} .
$$

On account of Lemma 1.1 and Hölder's inequality, we obtain similarly the inequality

$$
\left|\left(a_{0}(x, u), v\right)\right| \leqq\left\{C\|u\|_{p^{*}}\|v\|_{p}+\left(\|u\|_{(\alpha+1) p^{*}}\right)^{\alpha+1}\|v\|_{p}\right\} .
$$

By Poincaré's inequality, we get

$$
\begin{aligned}
& \|u\|_{p^{*}} \leqq C\|\nabla u\|_{p^{*}} \leqq C\|\nabla u\|_{p} \leqq C\|u\|_{V} \\
& \|v\|_{p} \leqq C\|\nabla v\|_{p} \leqq C\|v\|_{V} .
\end{aligned}
$$

And Sobolev's imbedding theorem yields the inequality

$$
\|u\|_{(\alpha+1) p^{*}} \leqq C\|u\|_{1, p}
$$

because $0 \leqq \alpha<n(p-1) /(n-p)-1$ for $p<n$. From (1.11)-(1.14) it follows that

$$
\left|\left(a_{0}(x, u), v\right)\right| \leqq C\left(\|u\|_{V}+\|u\|_{V}^{\alpha+1}\right)\|v\|_{V} .
$$

Combining (1.8), (1.10) with (1.15), we derive the following estimate from the definition of the dual norm of $A(u)$ in $V^{\prime}$ :

$$
\|A(u)\|_{V^{\prime}} \leqq C\left(\|u\|_{V}^{p-1}+\|u\|_{V}^{\alpha+1}+\|u\|_{V}\right)
$$

(ii) Hemi-continuity.

Let us suppose that $|\lambda| \leqq \lambda_{0}$. For $u, v$ and $w \in V$, it is valid that

$$
(A(u+\lambda v), w)=\int_{\Omega}\left\{\sum_{j=1}^{n} a_{j}(x, \nabla(u+\lambda v)) \partial_{x_{j}} w+a_{0}(x, u+\lambda v) w\right\} d x
$$

by the definition of the operator $A$. Applying the inequalities (1.1) and (1.2) to each term of the integrand in (1.17), we have 


$$
\begin{aligned}
& \left|a_{j}(x, \nabla(u+\lambda v)) \partial_{x_{j}} w\right| \leqq C\left\{\kappa_{0}\left(|\nabla u|+\lambda_{0}|\nabla v|\right)+|\nabla u|^{p-1}+\lambda_{0}^{p-1}|\nabla v|^{p-1}\right\}|\nabla w|, \\
& \left|a_{0}(x, u+\lambda v) w\right| \leqq C\left\{\left(|u|+\lambda_{0}|v|\right)+|u|^{\alpha+1}+\lambda_{0}^{\alpha+1}|v|^{\alpha+1}\right\}|w| .
\end{aligned}
$$

By the same way as in the proof of (i), we can prove that each term on the right-hand sides is integrable. Hence $(A(u+\lambda v), w)$ is continuous with respect to $\lambda$ by Lebesgue's convergence theorem.

(iii) Monotonicity.

From the remark after Lemma 1.1, it holds for any $u, v \in V$ that

$$
\begin{aligned}
(A(u)-A(v), u-v) & \geqq \gamma_{0}\left(\kappa_{0}\|\nabla(u-v)\|_{2}^{2}+\|\nabla(u-v)\|_{p}^{p}\right) \\
& \geqq 0 .
\end{aligned}
$$

Consequently, we have shown the pseudo-monotonicity of the operator $A$.

Finally we prove that the operator $A$ is coercive. Setting $v=0$ in (1.18), we have

$$
(A(u), u) \geqq \gamma_{0}\left(\kappa_{0}\|\nabla u\|_{2}^{2}+\|\nabla u\|_{p}^{p}\right) .
$$

Hence

$$
\frac{(A(u), u)}{\|u\|_{V}} \geqq C \gamma_{0}\|u\|_{V}^{p-1} \longrightarrow \infty \quad \text { as }\|u\|_{V} \longrightarrow \infty .
$$

Therefore the operator $A$ is coercive.

Q.E.D.

As we have mentioned at the beginning of this section, a solution of the variational inequality (3) exists for any $f \in V^{\prime}$ (J. L. Lions [29, p. 247]). The uniqueness of solutions follows immediately from (1.18).

The estimate for the gradient of the solution $u$ is carried out in the next lemma.

LEMmA 1.3. Under the assumption A if $f \in L^{p^{*}}(\Omega)$ (resp. $L^{2}(\Omega)$ ) and $\kappa_{0}$ $=0$ (resp. $\kappa_{0} \neq 0$ ), the gradient of the solution $u$ of the variational inequality (3) is estimated as follows:

$$
\kappa_{0}\|\nabla u\|_{2}^{2}+\|\nabla u\|_{p}^{p} \leqq C\left(1+\|f\|_{p^{*}}^{p^{*}}\right) \quad\left(\text { resp. } C\left(1+\|f\|_{2}^{2}\right)\right) .
$$

Proof. We denote by $u_{0}$ the particular solution of (3) for $f=0$. We easily see that

$$
\begin{aligned}
& \sum_{j=1}^{n}\left\langle a_{j}(x, \nabla u)-a_{j}\left(x, \nabla u_{0}\right), \partial_{x_{j}}\left(u-u_{0}\right)\right\rangle+\left(a_{0}(x, u)-a_{0}\left(x, u_{0}\right), u-u_{0}\right) \\
& \leqq\left(f, u-u_{0}\right) .
\end{aligned}
$$

Applying the inequalities (1.4) and (1.7) to the left-hand side, we get 


$$
\gamma_{0}\left(\kappa_{0}\left\|\nabla u-\nabla u_{0}\right\|_{2}^{2}+\left\|\nabla u-\nabla u_{0}\right\|_{p}^{p}\right) \leqq\left|\left(f, u-u_{0}\right)\right| .
$$

Using Hölder's inequality and Poincaré's inequality for the right-hand side of (1.21), we obtain the estimate (1.19).

Q.E.D.

We give a sufficient condition to assure that a sequence of functions converges weakly in $L^{q}(\Omega)$.

Lemma 1.4. Let $u$ be a distribution in $\Omega$ and let $\left\{u_{\nu}\right\}_{\nu=1}^{\infty}$ be a sequence in $L^{q}(\Omega)(1<q<\infty)$ such that the norms $\left\|u_{\nu}\right\|_{q}$ are uniformly bounded. If for any $\phi \in C_{0}^{\infty}(\Omega)$,

$$
\left(u_{\nu}, \phi\right) \longrightarrow(u, \phi) \quad \text { as } \nu \longrightarrow \infty,
$$

then $u$ belongs to $L^{q}(\Omega)$ and the sequence $u_{\nu}$ converges weakly to $u$ in $L^{q}(\Omega)$.

Proof. From the assumption it holds for any $\phi \in C_{0}^{\infty}(\Omega)$,

$$
|(u, \phi)| \leqq\left(\varlimsup_{\nu \rightarrow \infty}\left\|u_{\nu}\right\|_{q}\right)\|\phi\|_{q^{*}}{ }^{4)}
$$

For arbitrary $v \in L^{q^{*}}(\Omega)$ we take a sequence $\left\{\phi_{k}\right\}_{k=1}^{\infty}$ in $C_{0}^{\infty}(\Omega)$ such that $\left\|\phi_{k}-v\right\|_{q^{*}} \rightarrow 0$ as $k \rightarrow \infty$, then from (1.22) the sequence $\left\{\left(u, \phi_{k}\right)\right\}_{k=1}^{\infty}$ is a Cauchy sequence. Accordingly, $\lim _{k \rightarrow \infty}\left(u, \phi_{k}\right)$ exists and from (1.22) it is trivial that the limit depends only on $v$ and does not depend on any choice of the sequence. Hence we can express $\lim _{k \rightarrow \infty}\left(u, \phi_{k}\right)=l_{u}(v)$. It is easy to verify that $l_{u}$ is a linear functional on $L^{q^{*}}(\Omega)$, so there exists an element $L_{u}$ in $L^{q}(\Omega)\left(=\left(L^{q^{*}}(\Omega)\right)^{\prime}\right)$ such that $l_{u}(v)=\left(L_{u}, v\right)$ for all $v \in L^{q^{*}}(\Omega)$. The definition of $l_{u}(v)$ ensures the equality $(u, \phi)=\left(L_{u}, \phi\right)$ for any function $\phi$ in $C_{0}^{\infty}(\Omega)$, which is dense in $L^{q^{*}}(\Omega)$. Thus we can conclude that $u=L_{u}$ and therefore $u \in L^{q}(\Omega)$. The remainder of the proof is due to Theorem 3 in $[44$, p. 121].

Q.E.D.

2.

We introduce a coordinate transformation with the weight function $\Phi(x)$ and prepare some results with respect to it. We refer to some lemmas in [20].

Let $\boldsymbol{h}$ be a non-zero vector in $R^{n}$ with the length $h=|\boldsymbol{h}|$. Hereafter $h$ is assumed to be sufficiently small. As mentioned in the introduction we put $\Phi(x)=\operatorname{dis}(x, \partial \Omega \cup E)($ resp. $\operatorname{dis}(x, \partial \Omega))$ for $K=K_{1}\left(\operatorname{resp} . K=K_{2}\right)$ and we consider the transformation of the coordinates:

4) $q^{*}$ is the dual number of $q$, i.e., $q^{*}=q /(q-1)$. 


$$
\Phi_{h}: y=x+\Phi(x) h .
$$

We write $\boldsymbol{h}=\left(h_{1}, h_{2}, \cdots, h_{n}\right)$ and $\boldsymbol{J}=\partial\left(y_{1}, y_{2}, \cdots, y_{n}\right) / \partial\left(x_{1}, x_{2}, \cdots, x_{n}\right)$, then we have

$$
\boldsymbol{J}=\left[\begin{array}{cccc}
1+h_{1} \partial_{x_{1}} \Phi & h_{2} \partial_{x_{1}} \Phi & \cdots & h_{n} \partial_{x_{1}} \Phi \\
h_{1} \partial_{x_{2}} \Phi & 1+h_{2} \partial_{x_{2}} \Phi & \cdots & h_{n} \partial_{x_{2}} \Phi \\
\vdots & \vdots & \ddots & \vdots \\
h_{1} \dot{\partial}_{x_{n}} \Phi & h_{2} \partial_{x_{n}} \Phi & \cdots 1+h_{n} \partial_{x_{n}} \Phi
\end{array}\right]
$$

Let us put $\boldsymbol{e}=h^{-1} \boldsymbol{h}$ and let $\boldsymbol{e}$ be arbitrarily fixed. Noting that $\left|\partial_{x_{j}} \Phi\right|$ $\leqq 1$ ([20, p. 57]), we see that the determinant $J$ of $J$ is not zero for sufficiently small $h$, therefore the mapping $\Phi_{h}$ and its inverse $\Phi_{h}^{-1}$ are both one-to-one from $R^{n}$ onto itself. If we set $\Psi(y)=-\Phi(x)\left(=-\Phi\left(\Phi_{h}^{-1}(y)\right)\right)$, it is written

$$
\Phi_{h}^{-1}: x=y+\Psi(y) h .
$$

Here we remark that from (2.2) we can put $J=1+h J_{1}$ and the determinant $J^{-1}$ of the Jacobian $J^{-1}$ connected with the inverse transformation $\Phi_{h}^{-1}$ can be described in the form $J^{-1}=1+h J_{2}$, where $J_{1}$ and $J_{2}$ are uniformly bounded in $x \in \Omega$ and $h \in R^{n}$. Furthermore the transformation $\Phi_{h}$ maps $x \in \Omega$ to $y \in \Omega$ and $x \in \Omega^{c}$ to $y \in \Omega^{c}$ respectively, so it is a one-to-one mapping from $\Omega$ onto itself.

Now we define

$$
\left\{\begin{array}{l}
\left(S_{h} u\right)(x)=u(x+\Phi(x) h),\left(T_{h} u\right)(y)=u(y+\Psi(y) h), \\
\left(P_{h} u\right)(x)=h^{-1}\left[\left(S_{h} u\right)(x)-u(x)\right] \\
\left(Q_{h} u\right)(y)=h^{-1}\left[\left(T_{h} u\right)(y)-u(y)\right] .
\end{array}\right.
$$

Hereafter we write simply by $S_{h} u, T_{h} u, \ldots$ the functions $\left(S_{h} u\right)(x),\left(T_{h} u\right)(y)$, $\cdots$, respectively.

LEMMA 2.1 ([20, p. 58, 59]).

(i) We have

$$
\left\{\begin{array}{l}
\nabla_{x}\left(S_{h} u\right)=S_{h} \nabla_{x} u+h\left(e \cdot S_{h} \nabla_{x} u\right) \nabla_{x} \Phi, \\
\nabla_{y}\left(T_{h} u\right)=T_{h} \nabla_{y} u+h\left(e \cdot T_{h} \nabla_{y} u\right) \nabla_{y} \Psi, \\
\nabla_{x}\left(P_{h} u\right)=P_{h} \nabla_{x} u+\left(e \cdot S_{h} \nabla_{x} u\right) \nabla_{x} \Phi, \\
\nabla_{y}\left(Q_{h} u\right)=Q_{h} \nabla_{y} u+\left(e \cdot T_{h} \nabla_{y} u\right) \nabla_{y} \Psi .
\end{array}\right.
$$

(ii) If $u \in W^{1, q}(\Omega)(1<q<\infty)$, there exists a constant $C$ independent of $\boldsymbol{h}$ and $u$ such that 


$$
\left\|P_{h} u\right\|_{q}, \quad\left\|Q_{h} u\right\|_{q} \leqq C\|\nabla u\|_{q} .
$$

We do not repeat the proof of the above lemma, since it is parallel to that of [20].

The following lemma is as important as Lemma 1.4 for the proof of our theorem.

Lemma 2.2. If $u \in L^{q}(\Omega)(1<q<\infty)$, then for any function $\phi$ in $C_{0}^{\infty}(\Omega)$ it holds that

$$
\left(P_{h} u-J_{2} u, \phi\right) \longrightarrow((e \cdot \nabla)(\Phi u), \phi) \quad \text { as } h \longrightarrow 0,
$$

where the derivative $(\boldsymbol{e} \cdot \nabla)(\Phi u)$ of $\Phi u$ is in the sense of the distribution.

Proof. For any $u \in L^{q}(\Omega)$ and $\phi \in L^{q^{*}}(\Omega)$ the formula

$$
\left(P_{h} u, \phi\right)-\left(J_{2} u, \phi\right)=-\left(S_{h} u, P_{h} \phi\right)
$$

holds. The function $\phi$ belongs to $C_{0}^{\infty}(\Omega)$ in this case, so we can prove the following convergence by the similar technique as in [20] (Lemma 5 in p. 59):

$$
P_{h} \phi \longrightarrow \Phi(e \cdot \nabla) \phi \quad \text { in } L^{q^{*}}(\Omega) \quad \text { as } h \longrightarrow 0 .
$$

On the other hand it is an immediate consequence that

$$
S_{h} u \longrightarrow u \text { in } L^{q}(\Omega) \text { as } h \longrightarrow 0,
$$

from the fact that $u \in L^{q}(\Omega)$. Therefore the right-hand side on (2.8) tends to $-(u, \Phi(e \cdot \nabla) \phi)$ by virtue of $(2.9)$ and (2.10). In this way we arrive at the assertion of this lemma.

Q.E.D.

3.

This section is devoted to the statement and the proof of the main proposition in this paper. It is very important for the proof of our theorem.

Proposition. Let us assume the assumption A. Then the following estimates hold for the solution $u$ of the variational inequality (3):

(i) If $\kappa_{0}=0$ and $f \in W^{1, p^{*}}(\Omega)$,

$$
\left\|\left|S_{h} \nabla u\right|^{(p-2) / 2} P_{h} \nabla u\right\|_{2}^{2}+\left\||\nabla u|^{(p-2) / 2} P_{h} \nabla u\right\|_{2}^{2} \leqq C\left(1+\|f\|_{1, p^{*}}^{p^{*}}+\|f\|_{p^{*}}^{(\alpha+2) /(p-1)}\right) .
$$

(ii) If $\kappa_{0} \neq 0$ and $f \in L^{2}(\Omega)$,

$$
\left\|P_{h} \nabla u\right\|_{2}^{2}+\left\|\left|S_{h} \nabla u\right|^{(p-2) / 2} P_{h} \nabla u\right\|_{2}^{2}+\left\|\left.|| \nabla u\right|^{(p-2) / 2} P_{h} \nabla u\right\|_{2}^{2} \leqq C\left(1+\|f\|_{2}^{2 \beta / p}\right),
$$


where $\beta=\max (p, \alpha+2)$.

Proof. From now on we write simply $\partial_{x_{j}}=\partial_{j}$ or $\partial_{y_{j}}=\partial_{j}$ and we abbreviate the notation of sums.

As $S_{h} u$ and $T_{h} u$ belong to $K_{i}(i=1$ or 2) (see p. 60 in [20]), so we can put $v=S_{h} u$ and $T_{h} u$ in (3). Hence we obtain the inequalities

$$
\begin{aligned}
& \left\langle a_{j}(x, \nabla u), \partial_{j}\left(u-S_{h} u\right)\right\rangle+\left(a_{0}(x, u), u-S_{h} u\right) \leqq\left(f, u-S_{h} u\right), \\
& \left\langle a_{j}(y, \nabla u), \partial_{j}\left(u-T_{h} u\right)\right\rangle+\left(a_{0}(y, u), u-T_{h} u\right) \leqq\left(f, u-T_{h} u\right) .
\end{aligned}
$$

Adding these two inequalities, we have

$$
\begin{aligned}
& \left\langle a_{j}(x, \nabla u), \partial_{j}\left(u-S_{h} u\right)\right\rangle+\left\langle a_{j}(y, \nabla u), \partial_{j}\left(u-T_{h} u\right)\right\rangle \\
& \quad+\left(a_{0}(x, u), u-S_{h} u\right)+\left(a_{0}(y, u), u-T_{h} u\right) \\
& \quad \leqq\left(f, u-S_{h} u\right)+\left(f, u-T_{h} u\right) .
\end{aligned}
$$

Denoting each term on the left-hand side by $I_{j}(j=1,2,3,4)$ in turn from the left, we write $I_{1}$ and $I_{2}$ as follows by Lemma 2.1:

$$
\begin{aligned}
& I_{1}=\left\langle a_{j}(x, \nabla u), \partial_{j} u-S_{h} \partial_{j} u\right\rangle-h\left\langle a_{j}(x, \nabla u),\left(\boldsymbol{e} \cdot S_{h} \nabla u\right) \partial_{j} \Phi\right\rangle, \\
& I_{2}=\left\langle\left(1+h J_{1}\right) S_{h} a_{j}(x, \nabla u), S_{h} \partial_{j} u-\partial_{j} u\right\rangle-h\left\langle a_{j}(y, \nabla u),\left(\boldsymbol{e} \cdot T_{h} \nabla u\right) \partial_{j} \Psi\right\rangle .
\end{aligned}
$$

Consequently, we get

$$
\begin{aligned}
I_{1}+I_{2} & =\left\langle S_{h} a_{j}-a_{j}, S_{h} \partial_{j} u-\partial_{j} u\right\rangle+h\left\langle J_{1} S_{h} a_{j}, S_{h} \partial_{j} u-\partial_{j} u\right\rangle \\
& -h\left\langle a_{j},\left(e \cdot T_{h} \nabla u\right) \partial_{j} \Psi\right\rangle-h\left\langle a_{j},\left(e \cdot S_{h} \nabla u\right) \partial_{j} \Phi\right\rangle .
\end{aligned}
$$

After putting the right-hand side of (3.2) by $\sum_{j=5}^{8} I_{j}$, we estimate each term $I_{j}$. We rewrite $I_{5}$ in the form

$$
\begin{aligned}
I_{5} & =\left\langle a_{j}\left(S_{h} x, S_{h} \nabla u\right)-a_{j}\left(x, S_{h} \nabla u\right), S_{h} \partial_{j} u-\partial_{j} u\right\rangle \\
& +\left\langle a_{j}\left(x, S_{h} \nabla u\right)-a_{j}(x, \nabla u), S_{h} \partial_{j} u-\partial_{j} u\right\rangle .
\end{aligned}
$$

For the first term in (3.3), the following estimate holds:

$$
\begin{aligned}
& \left|\left\langle a_{l}\left(S_{h} x, S_{h} \nabla u\right)-a_{j}\left(x, S_{h} \nabla u\right), S_{h} \partial_{j} u-\partial_{j} u\right\rangle\right| \\
& \quad \leqq C h^{2} \int_{\Omega}\left(\kappa_{0}\left|S_{h} \nabla u\right| \cdot\left|P_{h} \nabla u\right|+\left|S_{h} \nabla u\right|^{p-1}\left|P_{h} \nabla u\right|\right) d x \\
& \quad \leqq C h^{2}\left(\kappa_{0}\|\nabla u\|_{2}\left\|P_{h} \nabla u\right\|_{2}+\|\nabla u\|_{p}^{p / 2}\left\|\left|S_{h} \nabla u\right|^{(p-2) / 2} P_{h} \nabla u\right\|_{2}\right) .
\end{aligned}
$$

Here we have used the equality

$$
a_{j}\left(S_{h} x, S_{h} \nabla u\right)-a_{j}\left(x, S_{h} \nabla u\right)=\int_{0}^{1} \sum_{k=1}^{n} \frac{\partial a_{j}}{\partial x_{k}}\left(x+\theta \Phi(x) h, S_{h} \nabla u\right) h_{k} \Phi(x) d \theta
$$

and the inequality in the assumption $\mathrm{A}$. 
Secondly the remainding term in (3.3) is estimated from below by (1.3) as follows:

$$
\begin{aligned}
& \left\langle a_{j}\left(x, S_{h} \nabla u\right)-a_{j}(x, \nabla u), S_{h} \partial_{j} u-\partial_{j} u\right\rangle \\
& \quad \geqq h^{2} \gamma_{0}\left\{\kappa_{0}\left\|P_{h} \nabla u\right\|_{2}^{2}+\left\|\left|S_{h} \nabla u\right|^{(p-2) / 2} P_{h} \nabla u\right\|_{2}^{2}+\left\||\nabla u|^{(p-2) / 2} P_{h} \nabla u\right\|_{2}^{2}\right\} .
\end{aligned}
$$

In this way (3.3), (3.4) and (3.5) yield that

$$
\begin{aligned}
I_{5} \geqq & \frac{\gamma_{0} h^{2}}{2}\left\{\kappa_{0}\left\|P_{h} \nabla u\right\|_{2}^{2}+\left\|\left.S_{h} \nabla u\right|^{(p-2) / 2} P_{h} \nabla u\right\|_{2}^{2}+\left\|\left.\nabla u\right|^{(p-2) / 2} P_{h} \nabla u\right\|_{2}^{2}\right\} \\
& -C h^{2}\left(\kappa_{0}\|\nabla u\|_{2}^{2}+\|\nabla u\|_{p}^{p}\right) .
\end{aligned}
$$

Next we see by Lemma 1.1 that

$$
\begin{aligned}
\left|I_{6}\right| & \leqq C h^{2} \int_{\Omega}\left(\kappa_{0}+\left|S_{h} \nabla u\right|^{p-2}\right)\left|S_{h} \nabla u\right| \cdot\left|P_{h} \nabla u\right| d x \\
& \leqq C h^{2}\left\{\kappa_{0}\|\nabla u\|_{2}\left\|P_{h} \nabla u\right\|_{2}+\|\nabla u\|_{p}^{p / 2}\left\|\left|S_{h} \nabla u\right|^{(p-2) / 2} P_{h} \nabla u\right\|_{2}\right\} .
\end{aligned}
$$

Before proceeding to successive terms $I_{7}$ and $I_{8}$, we note that we can write $\nabla_{y} \Psi=\nabla_{x} \Psi\left({ }^{t} J^{-1}\right)=-\left(\nabla_{x} \Phi\right)(I+h H)$, where $I$ is the unit matrix and each component of the matrix $H$ is essentially bounded (see p. 64 in [20]). Consequently, it is seen that

$$
\begin{aligned}
I_{7}+I_{8} & =-h\left\{\left\langle a_{j},\left(\boldsymbol{e} \cdot T_{h} \nabla u\right)\left[\left(-\nabla_{x} \Phi(x)\right)(I+h H)\right]_{j}\right\rangle\right. \\
& \left.+\left\langle a_{j},\left(\boldsymbol{e} \cdot S_{h} \nabla u\right) \partial_{j} \Phi\right\rangle\right\}^{5)} \\
& =-h\left\{-\left\langle\left(1+h J_{1}\right) S_{h} a_{j},(\boldsymbol{e} \cdot \nabla u) \partial_{j} \Phi\right\rangle+\left\langle a_{j},\left(\boldsymbol{e} \cdot S_{h} \nabla u\right) \partial_{j} \Phi\right\rangle\right\} \\
& +h^{2}\left\langle a_{j},\left(\boldsymbol{e} \cdot T_{h} \nabla u\right)\left(\nabla_{x} \Phi H\right)_{j}\right\rangle \\
& =h\left\{\left\langle S_{h} a_{j},(\boldsymbol{e} \cdot \nabla u) \partial_{j} \Phi\right\rangle-\left\langle a_{j},\left(\boldsymbol{e} \cdot S_{h} \nabla u\right) \partial_{j} \Phi\right\rangle\right\} \\
& +h^{2}\left\langle J_{1} S_{h} a_{j},(\boldsymbol{e} \cdot \nabla u) \partial_{j} \Phi\right\rangle+h^{2}\left\langle a_{j},\left(\boldsymbol{e} \cdot T_{h} \nabla u\right)\left(\nabla_{x} \Phi H\right)_{j}\right\rangle .
\end{aligned}
$$

Again we write $I_{7}+I_{8}=\sum_{j=9}^{11} I_{\jmath}$ in (3.8). We rewrite $I_{9}$ in the same way as in (3.3), i.e.,

$$
\begin{aligned}
I_{9}= & h\left\langle a_{j}\left(S_{h} x, S_{h} \nabla u\right)-a_{j}\left(x, S_{h} \nabla u\right),(e \cdot \nabla u) \partial_{j} \Phi\right\rangle \\
& +h\left\langle a_{j}\left(x, S_{h} \nabla u\right)-a_{j}(x, \nabla u),(e \cdot \nabla u) \partial_{j} \Phi\right\rangle \\
& +h\left\langle a_{j}(x, \nabla u), e \cdot\left(\nabla u-S_{h} \nabla u\right) \partial_{j} \Phi\right\rangle .
\end{aligned}
$$

By applying the similar technique as in (3.4) to the first term of (3.9), we get the inequality

$$
\left.h K a_{j}\left(S_{h} x, S_{h} \nabla u\right)-a_{j}\left(x, S_{h} \nabla u\right),(e \cdot \nabla u) \partial_{j} \Phi\right\rangle \mid \leqq C h^{2}\left(\kappa_{0}\|\nabla u\|_{2}^{2}+\|\nabla u\|_{p}^{p}\right) .
$$

\footnotetext{
5) We write the $j$-th component of a vector $U$ by $U_{j}$.
} 
The second term of (3.9) is estimated as follows:

$$
\begin{aligned}
& h\left|\left\langle a_{j}\left(x, S_{h} \nabla u\right)-a_{j}(x, \nabla u),(\boldsymbol{e} \cdot \nabla u) \partial_{j} \Phi\right\rangle\right| \\
& \quad \leqq C h^{2} \int_{\Omega}\left(\kappa_{0}+|\nabla u|^{p-2}+\left|S_{h} \nabla u\right|^{p-2}\right)\left|P_{h} \nabla u\right| \cdot|\nabla u| d x \\
& \quad \leqq C h^{2}\left\{\kappa_{0}\|\nabla u\|_{2}\left\|P_{h} \nabla u\right\|_{2}+\|\nabla u\|_{p}^{p / 2}\left(\left\||\nabla u|^{(p-2) / 2} P_{h} \nabla u\right\|_{2}\right.\right. \\
& \left.\left.\quad+\left\|\left|S_{h} \nabla u\right|^{(p-2) / 2} P_{h} \nabla u\right\|_{2}\right)\right\} .
\end{aligned}
$$

In the above estmiates we have used the assumption $\mathrm{A}$ and the equality

$$
a_{j}\left(x, S_{h} \nabla u\right)-a_{j}(x, \nabla u)=\int_{0}^{1} \sum_{i=1}^{n} \frac{\partial a_{j}}{\partial \eta_{i}}\left(x, \theta S_{h} \nabla u+(1-\theta) \nabla u\right)\left(S_{h} \partial_{i} u-\partial_{i} u\right) d \theta
$$

According to Lemma 1.1 the last term of (3.9) is estimated as follows:

$$
\begin{gathered}
h\left\langle\left\langle a_{j}, \boldsymbol{e} \cdot\left(\nabla u-S_{h} \nabla u\right) \partial_{j} \Phi\right\rangle\left|\leqq C h^{2} \int_{\Omega}\left(\kappa_{0}+|\nabla u|^{p-2}\right)\right| \nabla u|\cdot| P_{h} \nabla u\right| d x \\
\leqq C h^{2}\left(\kappa_{0}\|\nabla u\|_{2}\left\|P_{h} \nabla u\right\|_{2}+\|\nabla u\|_{p}^{p / 2}\left\||\nabla u|^{(p-2) / 2} P_{h} \nabla u\right\|_{2}\right) .
\end{gathered}
$$

By virtue of (3.9)-(3.12) we get

$$
\begin{aligned}
\left|I_{9}\right| & \leqq C h^{2}\left\{\kappa_{0}\|\nabla u\|_{2}\left\|P_{h} \nabla u\right\|_{2}+\kappa_{0}\|\nabla u\|_{2}^{2}+\|\nabla u\|_{p}^{p}\right. \\
& \left.+\|\nabla u\|_{p}^{p / 2}\left(\left\|\left.\nabla u\right|^{(p-2) / 2} P_{h} \nabla u\right\|_{2}+\left\|\left|S_{h} \nabla u\right|^{(p-2) / 2} P_{h} \nabla u\right\|_{2}\right)\right\} .
\end{aligned}
$$

On the other hand by Lemma 1.1, $I_{10}$ and $I_{11}$ are immediately estimated, that is,

$$
\left|I_{10}\right|,\left|I_{11}\right| \leqq C h^{2}\left(\kappa_{0}\|\nabla u\|_{2}^{2}+\|\nabla u\|_{p}^{p}\right) .
$$

Consequently (3.8), (3.13) and (3.14) yield that

$$
\begin{aligned}
\mid I_{7}+I_{3} ! & \leqq C h^{2}\left\{\kappa_{0}\left(\|\nabla u\|_{2}\left\|P_{h} \nabla u\right\|_{2}+\|\nabla u\|_{2}^{2}\right)+\|\nabla u\|_{p}^{p}\right. \\
& \left.+\|\nabla u\|_{p}^{p / 2}\left(\left\|\left.\nabla u\right|^{(p-2) / 2} P_{h} \nabla u\right\|_{2}+\left\|\left|S_{h} \nabla u\right|^{(p-2) / 2} P_{h} \nabla u\right\|_{2}\right)\right\} .
\end{aligned}
$$

With the aid of (3.2), (3.6), (3.7) and (3.15) we obtain the inequality

$$
\begin{aligned}
I_{1}+I_{2} & \geqq \frac{\gamma_{0} h^{2}}{4}\left\{\kappa_{0}\left\|P_{h} \nabla u\right\|_{2}^{2}+\left\|\left|S_{h} \nabla u\right|^{(p-2) / 2} P_{h} \nabla u\right\|_{2}^{2}\right. \\
& \left.+\left\|\left.\nabla u\right|^{(p-2) / 2} P_{h} \nabla u\right\|_{2}^{2}\right\}-C h^{2}\left(\kappa_{0}\|\nabla u\|_{2}^{2}+\|\nabla u\|_{p}^{p}\right) .
\end{aligned}
$$

Now we estimate the sum of $I_{3}$ and $I_{4}$. It is written in the form

$$
\begin{aligned}
I_{3}+I_{4} & =\left(a_{0}, u-S_{h} u\right)+\left(\left(1+h J_{1}\right) S_{h} a_{0}, S_{h} u-u\right) \\
& =\left(a_{0}\left(S_{h} x, S_{h} u\right)-a_{0}\left(x, S_{h} u\right), S_{h} u-u\right) \\
& +\left(a_{0}\left(x, S_{h} u\right)-a_{0}(x, u), S_{h} u-u\right)+h\left(J_{1} S_{h} a_{0}, S_{h} u-u\right) .
\end{aligned}
$$


Similarly as in (3.4) we can estimate the first term of (3.17) as follows:

$$
\begin{aligned}
& \left|\left(a_{0}\left(S_{h} x, S_{h} u\right)-a_{0}\left(x, S_{h} u\right), S_{h} u-u\right)\right| \\
& \quad \leqq C h^{2} \int_{\Omega}\left(1+\left|S_{h} u\right|^{\alpha+1}\right)\left|P_{h} u\right| d x \\
& \quad \leqq C h^{2}\left\{\left\|P_{h} u\right\|_{p}+\left\|P_{h} u\right\|_{p}\left(\|u\|_{(\alpha+1) p^{*}}\right)^{\alpha+1}\right\} \\
& \quad \leqq C h^{2}\left(\|\nabla u\|_{p}+\|\nabla u\|_{p}^{\alpha+2}\right) .
\end{aligned}
$$

Here we have used Lemma 2.1 and (1.14).

Because of Lemma 1.1 the second term of (3.17) is non-negative, i.e.,

$$
\left(a_{0}\left(x, S_{h} u\right)-a_{0}(x, u), S_{h} u-u\right) \geqq 0 .
$$

For the last term in (3.17) we attain the following estimate from Lemma 1.1 and Hölder's inequality:

$$
\begin{gathered}
h\left|\left(J_{1} S_{h} a_{0}, S_{h} u-u\right)\right| \leqq C h^{2} \int_{\Omega}\left(1+\left|S_{h} u\right|^{\alpha}\right)\left|S_{h} u\right| \cdot\left|P_{h} u\right| d x \\
\leqq C h^{2}\left\{\left\|P_{h} u\right\|_{p}\left\|S_{h} u\right\|_{p^{*}}+\left\|P_{h} u\right\|_{p}\left(\left\|S_{h} u\right\|_{(\alpha+1) p^{*}}\right)^{\alpha+1}\right\} .
\end{gathered}
$$

Similarly as in (3.18) we obtain the following inequality from (3.20):

$$
h\left|\left(J_{1} S_{h} a_{0}, S_{h} u-u\right)\right| \leqq C h^{2}\left(\|\nabla u\|_{p}^{2}+\|\nabla u\|_{p}^{\alpha+2}\right) .
$$

In this way we arrive at the inequality

$$
I_{3}+I_{4} \geqq-C h^{2}\left(\|\nabla u\|_{p}^{\alpha+2}+\|\nabla u\|_{p}+\|\nabla u\|_{p}^{2}\right)
$$

from (3.17), (3.18), (3.19) and (3.21).

Finally we estimate the right-hand side of (3.1). By simple calculations it holds that

$$
\left(f, u-T_{h} u\right)+\left(f, u-S_{h} u\right)=h^{2}\left\{\left(P_{h} f, P_{h} u\right)+\left(J_{1} S_{h} f, P_{h} u\right)\right\} .
$$

Let us suppose that $\kappa_{0}=0$. From (3.23) and (ii) in Lemma 2.1 we have

$$
\text { | the right-hand side of }(3.1) \mid \leqq C h^{2}\|\nabla u\|_{p}\|f\|_{1, p^{*}} \text {. }
$$

Combining (3.1), (3.16), (3.22) with (3.24), we deduce that if $\kappa_{0}=0$,

$$
\begin{aligned}
& \left\|\left.S_{h} \nabla u\right|^{(p-2) / 2} P_{h} \nabla u\right\|_{2}^{2}+\left\|\left.\nabla u\right|^{(p-2) / 2} P_{h} \nabla u\right\|_{2}^{2} \\
& \quad \leqq C\left(\|\nabla u\|_{p}^{2}+\|\nabla u\|_{p}^{\alpha+2}+\|\nabla u\|_{p}+\|\nabla u\|_{p}^{p}+\|f\|_{1, p^{*}}^{p^{*}}\right) .
\end{aligned}
$$

Therefore if $\kappa_{0}=0$, our proposition is correct in virtue of Lemma 1.3.

Secondly we suppose that $\kappa_{0} \neq 0$. In this case we transform the"first term in the brackets on the right-hand side of (3.23) into the following 
form by the expression (2.8) in the proof of Lemma 2.2:

$$
\left(P_{h} f, P_{h} u\right)=-\left(S_{h} f, P_{h} P_{h} u\right)+\left(J_{2} f, P_{h} u\right) .
$$

Taking account of (3.23) and (ii) in Lemma 2.1, we obtain the inequality

$$
\text { | the right-hand side of (3.1) } \mid \leqq C h^{2}\|f\|_{2}\left(\left\|P_{h} \nabla u\right\|_{2}+\|\nabla u\|_{2}\right) \text {. }
$$

Hence from (3.1), (3.16), (3.22) and (3.25) it follows that

$$
\begin{aligned}
\gamma_{0}\left\{\kappa_{0} \|\right. & \left.\left.P_{h} \nabla u\left\|_{2}^{2}+\right\|\left|S_{h} \nabla u\right|^{(p-2) / 2} P_{h} \nabla u\left\|_{2}^{2}+\right\| \nabla u\right|^{(p-2) / 2} P_{h} \nabla u \|_{2}^{2}\right\} \\
& \leqq C\left(\kappa_{0}\|\nabla u\|_{2}^{2}+\|\nabla u\|_{p}^{p}+\|\nabla u\|_{p}^{\alpha+2}+\|\nabla u\|_{p}+\|\nabla u\|_{p}^{2}+\|f\|_{2}^{2}\right) .
\end{aligned}
$$

Thus the proof of our proposition is accomplished by the straight-forward application of Lemma 1.3 .

Q.E.D.

4.

In this section we prove our theorem.

At first we show that $\Phi(x) a_{j}(x, \nabla u)$ belongs to $W^{1, p^{*}}(\Omega)$ and we estimate its $W^{1, p^{*}}(\Omega)$ norm. Considering Lemma 1.4 and Lemma 2.2, we first give the uniform $L^{p^{*}}(\Omega)$ estimate for the sequence $\left\{P_{h}\left(a_{j}(x, \nabla u)\right)-J_{2} a_{j}(x, \nabla u)\right\}_{h>0}$, where $h$ is sufficiently small. We write $P_{h}\left(a_{j}(x, \nabla u)\right)$ in the form

$$
\begin{aligned}
P_{h}\left(a_{j}(x, \nabla u)\right) & =\left[a_{j}\left(S_{h} x, S_{h} \nabla u\right)-a_{j}\left(x, S_{h} \nabla u\right)\right] h^{-1} \\
& +\left[a_{j}\left(x, S_{h} \nabla u\right)-a_{j}(x, \nabla u)\right] h^{-1} .
\end{aligned}
$$

Similarly as in (3.4) we see that

$$
\left\|\left[a_{j}\left(S_{h} x, S_{h} \nabla u\right)-a_{j}\left(x, S_{h} \nabla u\right)\right] h^{-1}\right\|_{p^{*}}^{p^{*}} \leqq C\left(\kappa_{0}^{p^{*}}\|\nabla u\|_{p^{*}}^{p^{*}}+\|\nabla u\|_{p}^{p}\right) .
$$

For the second term of (4.1), we get the inequality

$$
\begin{aligned}
& \left\|\left[a_{j}\left(x, S_{h} \nabla u\right)-a_{j}(x, \nabla u)\right] h^{-1}\right\|_{p^{*}}^{p^{*}} \\
& \quad \leqq C\left\{\kappa_{0}^{p^{*}}\left\|P_{h} \nabla u\right\|_{p^{*}}^{p^{*}}+\int_{\Omega}\left(\left|P_{h} \nabla u\right|^{p^{*}}\left|S_{h} \nabla u\right|^{p^{*(p-2)}}+\left|P_{h} \nabla u\right|^{\left.\left.p^{*}|\nabla u|^{\left.p^{*(p-2)}\right)}\right) d x\right\}}\right.\right.
\end{aligned}
$$

by the same way as in (3.11).

Because of $1<p^{*} \leqq 2 \leqq p$, we have $\left\|P_{h} \nabla u\right\|_{p^{*}} \leqq C\left\|P_{h} \nabla u\right\|_{2}$. And the integral terms are estimated in the following way:

$$
\begin{aligned}
& \int_{\Omega}\left|P_{h} \nabla u\right|^{p^{*}}\left|S_{h} \nabla u\right|^{p^{*}(p-2)} d x \\
& \quad \leqq\left[\int_{\Omega}\left\{\left|P_{h} \nabla u\right|^{p^{*}}\left|S_{h} \nabla u\right|^{p^{*}(p-2) / 2}\right\}^{2 / p^{*}} d x\right]^{p^{*} / 2}\left[\int_{\Omega}\left|S_{h} \nabla u\right|^{\left(p^{*}(p-2) / 2\right)\left(1-p^{*} / 2\right)-1} d x\right]^{\left(1-p^{*} / 2\right)}
\end{aligned}
$$




$$
\begin{aligned}
& =\left(\int_{\Omega}\left|P_{h} \nabla u\right|^{2}\left|S_{h} \nabla u\right|^{p-2} d x\right)^{p^{*} / 2}\left(\int_{\Omega}\left|S_{h} \nabla u\right|^{p} d x\right)^{\left.\left(1-p^{*} / 2\right) 6\right)} \\
& \leqq C\left\{\left\|\left|S_{h} \nabla u\right|^{(p-2) / 2} P_{h} \nabla u\right\|_{2}^{2}+\|\nabla u\|_{p}^{p}\right\}
\end{aligned}
$$

where we use Hölder's inequality and Minkowski's inequality. Another integral term is similarly estimated.

Hence we conclude from (4.3) that

$$
\begin{aligned}
& \left\|\left[a_{\jmath}\left(x, S_{h} \nabla u\right)-a_{j}(x, \nabla u)\right] h^{-1}\right\|_{p^{*}}^{p^{*}} \\
& \quad \leqq C\left\{\kappa_{0}^{p *}\left\|P_{h} \nabla u\right\|_{2}^{p *}+\|\nabla u\|_{p}^{p}+\left(\left\|\left|S_{h} \nabla u\right|^{(p-2) / 2} P_{h} \nabla u\right\|_{2}^{2}+\left\||\nabla u|^{(p-2) / 2} P_{h} \nabla u\right\|_{2}^{2}\right)\right\} .
\end{aligned}
$$

Because of (4.1), (4.2) and (4.4) it holds that

$$
\begin{aligned}
\left\|P_{h}\left(a_{j}(x, \nabla u)\right)\right\|_{p^{*}}^{p^{*}} & \leqq C\left\{\kappa_{0}^{p^{*}}\left(\|\nabla u\|_{p^{*}}^{p^{*}}+\left\|P_{h} \nabla u\right\|_{2}^{p^{*}}\right)+\|\nabla u\|_{p}^{p}\right. \\
& \left.+\left(\left\|\left|S_{h} \nabla u\right|^{(p-2) / 2} P_{h} \nabla u\right\|_{2}^{2}+\left\|\left.\nabla u\right|^{(p-2) / 2} P_{h} \nabla u\right\|_{2}^{2}\right)\right\}
\end{aligned}
$$

Therefore if $\kappa_{0}=0$,

$$
\left\|P_{h}\left(a_{j}(x, \nabla u)\right)\right\|_{p^{*}}^{p^{*}} \leqq C\left(1+\|f\|_{1, p^{*}}^{p^{*}}+\|f\|_{p^{*}}^{(\alpha+2) /(p-1)}\right),
$$

from (4.5), Proposition and Lemma 1.3. We write the right-hand side by $C \gamma_{f}^{0}$. Moreover if $\kappa_{0} \neq 0$, we have similarly

$$
\left\|P_{h}\left(a_{j}(x, \nabla u)\right)\right\|_{p^{*}}^{p^{*}} \leqq C\left(1+\|f\|_{2}^{2 \beta / p}\right),
$$

where $\beta=\max (p, \alpha+2)$. The right-hand side is written by $C \gamma_{f}$.

On the other hand the functions $J_{2}$ are uniformly bounded in $x$ and h. Thus by Lemma 1.1

$$
\left\|J_{2} a_{j}(x, \nabla u)\right\|_{p^{*}}^{p^{*}} \leqq C\left(\kappa_{0}^{p^{*}}\|\nabla u\|_{p^{*}}^{p^{*}}+\|\nabla u\|_{p}^{p}\right) .
$$

Applying the estimate for $\nabla u$ in Lemma 1.3 to each term on the right-hand side of (4.6), the $L^{p^{*}}(\Omega)$ norms of the functions $J_{2} a_{j}(x, \nabla u)$ are estimated as follows: if $\kappa_{0}=0$ (resp. $\kappa_{0} \neq 0$ ),

$$
\left\|J_{2} a_{j}(x, \nabla u)\right\|_{p^{*}}^{p^{*}} \leqq C \gamma_{f}^{0} \quad\left(\text { resp. } C \gamma_{f}\right) \text {. }
$$

From the above the $L^{p^{*}}(\Omega)$ norms of the sequence $\left\{P_{h}\left(a_{j}(x, \nabla u)\right)-\right.$ $\left.J_{2} a_{j}(x, \nabla u)\right\}_{h>0}$ are estimated. That is, if $\kappa_{0}=0$ (resp. $\kappa_{0} \neq 0$ ),

$$
\left\|P_{h}\left(a_{j}(x, \nabla u)\right)-J_{2} a_{j}(x, \nabla u)\right\|_{p^{*}}^{p^{*}} \leqq C \gamma_{f}^{0} \quad\left(\operatorname{resp} . C \gamma_{f}\right) .
$$

Because $a_{j}(x, \nabla u) \in L^{p^{*}}(\Omega)$, we conclude on account of Lemma 2.2 that for any $\phi \in C_{0}^{\infty}(\Omega)$

\footnotetext{
6) Indeed, $\left(p^{*}(p-2) / 2\right)\left(1-p^{*} / 2\right)^{-1}=p$.
} 


$$
\begin{gathered}
\left(P_{h}\left(a_{j}(x, \nabla u)\right)-J_{2} a_{j}(x, \nabla u), \phi\right) \longrightarrow\left((e \cdot \nabla)\left(\Phi a_{j}(x, \nabla u)\right), \phi\right), \\
\text { as } h \longrightarrow 0 .
\end{gathered}
$$

Hence Lemma 1.4 yields that the distribution $(e \cdot \nabla)\left(\Phi a_{j}(x, \nabla u)\right)$ belongs to $L^{p^{*}}(\Omega)$ and that

$$
\begin{aligned}
P_{h}\left(a_{j}(x, \nabla u)\right)-J_{2} a_{j}(x, \nabla u) & \longrightarrow(e \cdot \nabla)\left(\Phi a_{j}(x, \nabla u)\right) \quad \text { in } L^{p^{*}}(\Omega), \\
\text { as } h \longrightarrow 0, &
\end{aligned}
$$

where "—" means the weak convergence.

Therefore from (4.7) and (4.8) we derive that if $\kappa_{0}=0\left(\right.$ resp. $\left.\kappa_{0} \neq 0\right)$,

$$
\left\|(\boldsymbol{e} \cdot \nabla)\left(\Phi a_{j}(x, \nabla u)\right)\right\|_{p^{*}}^{p^{*}} \leqq C \gamma_{f}^{0} \quad\left(\text { resp. } C \gamma_{f}\right) .
$$

The assertion with respect to $\Phi(x)|\nabla u|^{p / 2}$ can be treated more easily. By simple calculations we deduce the inequality

$$
\left|P_{h}\left(|\nabla u|^{p / 2}\right)\right| \leqq C\left|P_{h} \nabla u\right|\left(\left|S_{h} \nabla u\right|^{(p-2) / 2}+|\nabla u|^{(p-2) / 2}\right) .
$$

From this inequality and the estimate in Proposition we have for the case of $\kappa_{0}=0\left(\right.$ resp. $\left.\kappa_{0} \neq 0\right)$

$$
\left.\left\|P_{h}\left(|\nabla u|^{p / 2}\right)\right\|_{2}^{2} \leqq C \gamma_{f}^{0} \quad \text { (resp. } C \gamma_{f}\right) .
$$

Since the functions $J_{2}$ are uniformly bounded, we get from Lemma 1.3

$$
\left.\left\|J_{2}|\nabla u|^{p / 2}\right\|_{2}^{2} \leqq C \gamma_{f}^{0} \quad \text { (resp. } C \gamma_{f}\right) \text {, }
$$

if $\kappa_{0}=0$ (resp. $\kappa_{0} \neq 0$ ).

Thus the $L^{2}(\Omega)$ norms of the sequence $\left\{P_{h}\left(|\nabla u|^{p / 2}\right)-J_{2}|\nabla u|^{p / 2}\right\}_{h>0}$ are estimated as follows: if $\kappa_{0}=0$ (resp. $\left.\kappa_{0} \neq 0\right)$,

$$
\left\|P_{\boldsymbol{h}}\left(|\nabla u|^{p / 2}\right)-J_{2}|\nabla u|^{p / 2}\right\|_{2}^{2} \leqq C \gamma_{f}^{0} \quad\left(\text { resp. } C \gamma_{f}\right) .
$$

With the aid of a priori estimate (4.10) we can select a sequence $\left\{h_{\nu}\right\}_{\nu=1}^{\infty}$ with $h_{\nu} \rightarrow 0(\nu \rightarrow \infty)$ and choose a function $v \in L^{2}(\Omega)$ such that

$$
P_{h_{\nu}}\left(|\nabla u|^{p / 2}\right)-J_{2}|\nabla u|^{p / 2} \longrightarrow v \text { in } L^{2}(\Omega) \text { as } \nu \longrightarrow \infty,
$$

and besides if $\kappa_{0}=0$ (resp. $\kappa_{0} \neq 0$ ),

$$
\|v\|_{2}^{2} \leqq C \gamma_{f}^{0} \quad\left(\text { resp. } C \gamma_{f}\right) .
$$

By Lemma 2.2 we see that for any function $\phi$ in $C_{0}^{\infty}(\Omega)$

$$
\begin{aligned}
&\left(P_{h}\left(|\nabla u|^{p / 2}\right)-J_{2}|\nabla u|^{p / 2}, \phi\right) \longrightarrow\left((e \cdot \nabla)\left(\Phi|\nabla u|^{p / 2}\right), \phi\right) \\
& \text { as } h \longrightarrow 0 .
\end{aligned}
$$


From (4.11), (4.12) and (4.13) it holds that $v=(e \cdot \nabla)\left(\Phi|\nabla u|^{p / 2}\right)$ and if $\kappa_{0}=0$ (resp. $\kappa_{0} \neq 0$ ),

$$
\left.\left\|(\boldsymbol{e} \cdot \nabla)\left(\Phi|\nabla u|^{p / 2}\right)\right\|_{2}^{2} \leqq C \gamma_{f}^{0} \quad \text { (resp. } C \gamma_{f}\right) .
$$

By virtue of (4.9) and (4.14) we have proved the required for $\Phi(x) a_{j}(x, \nabla u)$ and $\Phi(x)|\nabla u|^{p / 2}$. And the proof of the part for $\Phi(x) \nabla u$ is left. We briefly explain it. On account of the estimate (ii) in Proposition, $\left\|P_{h} u\right\|_{1,2}$ are uniformly bounded in $h$, more precisely,

$$
\left\|P_{h} u\right\|_{1,2} \leqq C \gamma_{f}^{1 / 2},
$$

if $\kappa_{0} \neq 0$.

Accordingly there are a sequence $\left\{h_{\mu}\right\}_{\mu=1}^{\infty}$ with $h_{\mu} \rightarrow 0(\mu \rightarrow \infty)$ and an element $w$ in $W^{1,2}(\Omega)$, to which the sequence $P_{h_{\mu}} u$ converges weakly in $W^{1,2}(\Omega)$ as $\mu \rightarrow \infty$. On the other hand the sequence $P_{h_{\mu}} u$ converges strongly to $\Phi(e \cdot \nabla) u$ in $L^{2}(\Omega)$ as $\mu \rightarrow \infty$, which is shown by the same way as in Lemma 5 in [20]. Consequently we have that $w=\Phi(\boldsymbol{e} \cdot \nabla) u$ and

$$
\|\Phi(\boldsymbol{e} \cdot \nabla) u\|_{1,2} \leqq C \gamma_{f}^{1 / 2} .
$$

Thus the proof of our theorem is finished.

Q.E.D.

Remark. The same conclusion is obtained if we replace $a_{j}(x, \nabla u)$ by $|\nabla u|^{p-2} \partial_{x_{j}} u$ or $\left|\partial_{x_{j}} u\right|^{p-2} \partial_{x_{j}} u$ in our theorem.

\section{BIBLIOGRAPHY}

[1] A. A. Arkhipova, A problem with discontinuous obstruction for uniformly elliptic equation, Vestn. Lenigr. Univ., 19 (1974), 154-155.

[2] - On limiting smoothness of the solution of a problem with a two-side barrier, Vestn. Lenigr. Univ. Mat., 17 (1984), 5-9.

[ 3 ] H. Brézis-D. Kinderlehrer, The smoothness of solutions to nonlinear variational inequalities, Indiana Univ. Math. J., 17 (1974), 831-844.

[4] - - G. Stampacchia, Sur la régularité de la solution d'inéquations elliptiques, Bull. Soc. Math. France, 96 (1968), 153-180.

[5] K. C. Chang, The obstacle problem and partial differential equations with discontinuous nonlinearities, Comm. Pure Appl. Math., 38 (1980), 117-146.

[6] F. Cooper, Existence and regularity of solutions with bounded gradient of variational inequalities of a certain class, Quart. J. Math. Oxford, 26 (1975), 203-214.

[ 7 ] J. P. Dias, Une classe de problèmes variationnels non linéarires de type elliptique ou parabolique, Ann. Mat. Pura Appl., 92 (1972), 263-322.

[8] E. DiBenedetto, $C^{1+\alpha}$ local regularity of weak solutions of degenerate elliptic equations, Nonlinear Anal. T.M.A., 7 (1983), 827-850.

[9] A. Domarkas, Regularity of solutions of quasilinear elliptic equations with unilateral boundary conditions, Lithu. Math. J., 20 (1980), 8-13. 
[10] _- Unilateral problems for quasilinear elliptic equation, ibid., 21 (1981), 317327.

[11] L. C. Evans, A new proof of local $C^{1, \alpha}$ regularity for solutions of certain degenerate elliptic P.D.E., J. Differential Equations, 45 (1982), 356-373.

[12] J. Frehse, Two dimensional variational problems with thin obstacles, Math. Z., 143 (1975), 279-288.

[13] - On Signorini's problem and variational problems with thin obstacles, Ann. Scuola Norm. Sup. Pisa, 4 (1977), 343-362.

[14] - On the smoothness of solutions of variational inequalities with obstacles, Banach Center Publ., 10 (1983), 87-128.

[15] — - U. Mosco, Irregular obstacles and quasi-variational inequalities of stochastic impulse control, Ann. Scuola Norm. Sup. Pisa, 9 (1982), 105-157.

[16] M. G. Garroni, Regularity of a nonlinear variational inequality with obstacle on the boundary, B.U.M.I. Anal. Fun. Appl. Suppl., 1 (1980), 267-286.

[17] C. Gerhardt, Regularity of solutions of nonlinear variational inequalities, Arch. Rational Mech. Anal., 52 (1973), 389-393.

[18] —, Global $C^{1,1}$-regularity for solutions of quasilinear variational inequalities, ibid., 89 (1985), 83-92.

[19] M. Giaquinta-G. Modica, Regolarità Lipschtziana per la soluzione di alcuni problemi di minimo con vincola, Ann. Mat. Pura Appl., 106 (1975), 95-117.

[20] K. Hayasida-H. Nagase, On solutions of variational inequalities constrained on a subset of positive capacity, Nagoya Math. J., 97 (1985), 51-69.

[21] G. Huisken, $C^{1,1}$-regularity of solutions to variational inequalities, Proc. Centre Math. Anal. Austral. Nat. Univ., 5 (1984), 85-90.

[22] D. Kinderlehrer, The regularity of the solution to a certain variational inequality, Proc. Symp. Pure Appl. Math., 23, A.M.S. Providence.

[23] - Variational inequalities with lower dimensional obstacles, Israel J. Math., 16 (1971), 339-348.

[24] - The smoothness of the solution of the boundary obstacle problem, J. Math. Pures Appl., 60 (1980), 193-212.

[25] K. L. Kuttler Jr., Degenerate variational inequalities of evolution, Nonlinear Anal. T.M.A., 8 (1984), 837-850.

[26] J. L. Lewis, Regularity of the derivatives of solutions to certain degenerate elliptic equations, Indiana Univ. Math. J., 32 (1983), 849-858.

[27] H. Lewy-G. Stampacchia, On existence and smoothness of solutions of some noncoercive variational inequalities, Arch. Rational Mech. Anal., 41 (1971), 241-252.

[28] P. Lindqvist, On the growth of the solutions of the differential equation div. $\left(|\nabla u|^{p-2} \nabla u\right)=0$ in $n$-dim. space, J. Differential Equations, 58 (1985), 307-317.

[29] J. L. Lions, Quelques méthodes de résolution des problèmes aux limites nonlinéaries, Dunod Gautyier-Villars, 1969.

[30] T. H. Michel, Regularity for solutions to obstacle problems, Proc. Centre, Math. Anal. Austral. Nat. Univ., 8 (1984), 29-37.

[31] R. Schaaf, Regularity of solutions to linear and quasilinear variational inequalities with two obstacles, Analysis, 2 (1982), 337-345.

[32] G. Stampacchia, Regularity of solutions of some variational inequalities Proc. Symp. Pure Math., 18, A.M.S. Providence.

[33] P. Tolksdorf, Regularity for a more general class of quasilinear elliptic equation, J. Differential Equations, 51 (1984), 126-150.

[34] N. N. Ural'tseva, Regularity of the solutions of variational inequalities, J. Soviet Math., 3 (1975), 565-573.

[35] H. Beirão da Veiga, Proprietà di sommabilità e di limitatezza per soluzioni di 
disequazioni variazionali ellittiche, Rend. Sem. Nat. Padova, 46 (1971), 141-171.

[36] — Sur la régularité des solutions de l'équation div. $A(x, u, \nabla u)=B(x, u, \nabla u)$ avec des conditions aux limites unilatérales et mêlées, Ann. Mat. Pura Appl., 93 (1972), 173-230.

[37] -F. Conti, Equazioni ellittiche non lineari con ostacoli sottili applicazioni allo studio dei punti regolari, Ann. Scuola Norm. Sup. Pisa, 26 (1972), 533-562.

[38] G. H. Williams, Lipschitz continuous solutions for nonlinear obstacle problems, Math. Z., 154 (1977), 51-65.

[39] - Smooth solutions for variational inequalities with obstacles, Indiana Univ. Math. J., 31 (1982), 317-325.

[40] G. H. Yakovlev, A variational problem, Differential Equations, 5 (1969), 960-966.

[41] - The first boundary value problem for quasilinear elliptic equations of second order, Proc. Steklov Inst. Math., 117 (1972), 381-403.

[42] - Properties of solutions of a class of second-order quasilinear elliptic equations in divergence form, ibid., 131 (1974), 242-252.

[43] - Some properties of solutions of quasilinear elliptic equations, ibid., 134 (1975) , 441-458.

[44] K. Yosida, Functional analysis, Springer-Verlag, 1968.

Suzuka College of Technology

Suzuka 510-02

Japan 
